\title{
Rapid and Sensitive Analysis of Eight Polyphenols in Tobacco by Rapid Resolution Liquid Chromatogarphy
}

\author{
Fuwei Xie ${ }^{1,2}$, Ajuan Yu ${ }^{1}$, Dengke Hou ${ }^{1}$, Huimin Liu ${ }^{2}$, Li Ding ${ }^{2}$, Shusheng Zhang ${ }^{1 *}$ \\ ${ }^{1}$ Chemistry Department of Zhengzhou University, Zhengzhou, China \\ ${ }^{2}$ Zhengzhou Tobacco Research Institute of CNTC, Zhengzhou, China \\ E-mail: 'zsszz@126.com \\ Received September 5, 2011; revised October 15, 2011; accepted October 29, 2011
}

\begin{abstract}
A rapid resolution liquid chromatographic (RRLC) method for the determination of eight polyphenols in tobacco was developed. Polyphenols were extracted from tobacco samples by methanol/ water in an ultrasonic bath, then subjected to clean up by solid phase extraction. The separation was performed on a $50 \times 4.6 \mathrm{~mm}$, $1.8 \mu \mathrm{m}$ ZORBAX Eclipse XDB-C18 column. Compared with conventional HPLC method, the analysis time for eight polyphenols by RRLC method was reduced from 20 to 5 min without sacrificing resolution, and the sensitivity was improved. This method appears simple, accurate and precious. The relative standard deviations (RSD) of overall analysis procedure for eight tobacco polyphenols were less than $2 \%$ with the recoveries ranging from $94 \%$ to $107 \%$. This method could be applied to the rapid determination of major polyphenols in tobacco with satisfactory results.
\end{abstract}

Keywords: Solid Phase Extraction, RRLC, Tobacco, Polyphenols

\section{Introduction}

Polyphenols, including tannic, cumarine, flavonid, and derivatives of simple phenols, are secondary metabolities in tobacco plant. They play important roles on the growth of tobacco and the quality of tobacco leaf [1-3]. The research results showed that in flue-cued tobacco leaves chlorogenic acid, scopoletin and rutin are the major polyphenols [4], and their combustion could generate phenolic compounds considered as carcinogens [5]. Therefore, it can pose serious harm on consumers' health during smoking. To understand the polyphenol content in tobacco, polyphenol transfer during smoking and the relationship between polyphenol and smokers' health, it is necessary to develop a practical method for the determinations of polyphenols in tobacco.

To date, several analytical methods for the analysis of polyphenols in tobacco have been reported by means of spectrophotometry [6], gas chromatography (GC) or GCMS [7], high-performance liquid chromatography (HPLC) with chemiluminescence detection [8], ultraviolet detection (UVD) [9,10] and MS detection [11], and capillary electrophoresis (CE) method [12]. Among these methods, spectrophotometry is only used for the determination of the total polyphenols, GC and HPLC are the most pow- erful. However, GC cannot be used directly to determine polyphenols due to their poor volatility, high polarity and/or thermal instability. It needs time-consuming derivation. In contrast GC, HPLC is more effective and appropriate for the separation and determination of polyphenols. In order to obtain satisfactory separation of about 5 - 10 polyphenols by the conventional HPLC, the longer retention or analysis times between $20-30 \mathrm{~min}$ are required. Obviously, it cannot satisfy the requirement for the rapid analysis of high sample throughput. To solve this problem, various strategies aimed at increasing the speed and performance of chromatographic separation can be considered. Currently, the smaller stationary phase particles $(<2.0 \mu \mathrm{m})$, new bridging structure of stationary phase and high-pressure systems are commercially available. Using this new rapid resolution liquid chromatography (RRLC) technique, higher linear velocity, faster run time, higher sensitivity and resolution are achieved [13-15]. Thus, the aims of the present work are 1) to develop a practical extraction and clean-up procedure prior to the analysis of eight polyphenols (Figure 1) in tobacco; 2) to develop a rapid and sensitive method to the analysis of eight polyphenols; and 3) to determine the polyphenols in actual tobacco samples by the developed RRLC. 


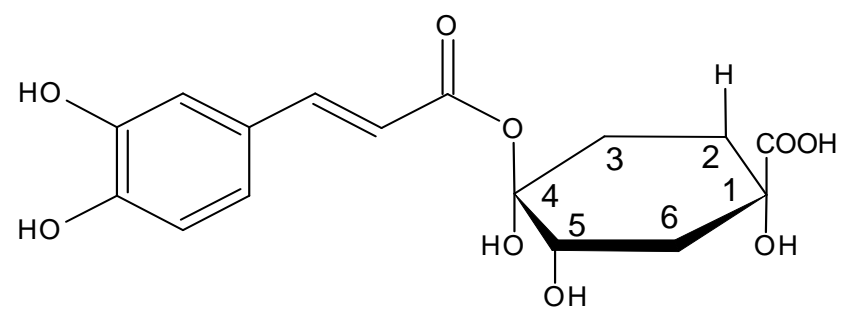

3-O-caffeoyl-d-quinic acid

Chlorogenic acid (3-O-caffeoyl-d-quinic acid)

Neo Chlorogenic acid (5-O-caffeoyl-d-quinic acid)<smiles>[R]c1cc(-c2oc3cc(O)cc(O)c3c(=O)c2OC)ccc1O</smiles>

Rutin

Quercitrin

Kaempferol-3-rutinoside

$$
\begin{array}{lll}
\mathrm{R}^{\prime}=\mathrm{OH} & \mathrm{R}^{\prime}=\text { Rutinosyl } & \text { Caffeic acid } \\
\mathrm{R}^{\prime}=\mathrm{OH} & \mathrm{R}^{\prime}=\mathrm{d}-\text { Glucosyl } & \\
\mathrm{R}^{\prime}=\mathrm{H} & \mathrm{R}^{\prime}=\text { l-Rhamnosido-d-Glucosyl R } &
\end{array}
$$<smiles>COc1cc2ccc(=O)oc2cc1O</smiles>

Scopoletin

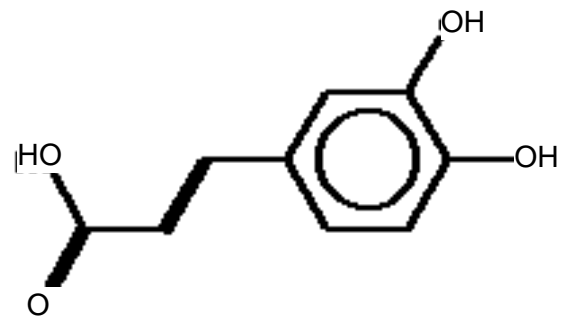

Figure 1. Structures for the eight polyphenols.

\section{Experimental}

\subsection{Reagents and Solution}

All solvent (HPLC grade) were purchased from J\&T Baker (Phillipsburg, NJ, USA), include methanol, acetonitrile and Formic acid. The certified standards used in this study were 5-O-caffeoylquinic acid (NG, 98\%), chlorogenic acid (CG, 98\%), 4-o-caffeoyl-quinic acid (YG, 98\%), caffeic acid (CA, 98\%), scopoletin (SP, 98\%), rutin(RT, 98\%), kaempferol-3-rutinoside (KR, 98\%) and quercitrin(QT, 98\%), respectively. They are obtained from Sigma-Aldrich, USA.

Stock solutions of polyphenols were prepared at 1000 $\mu \mathrm{g} / \mathrm{ml}$ in methanol and stored under refrigeration at $4^{\circ} \mathrm{C}$ in the dark. Quantification of samples was made using calibration curves of the eight polyphenols at the final concentration of $0.5,1.0,10,30,60$ and $120 \mu \mathrm{g} / \mathrm{mL}$ in the solution of methanol-water $(9: 1, \mathrm{v} / \mathrm{v})$. Each determination was performed in triplicate.

\subsection{Apparatus and RRLC Conditions}

Agilent 1200 RRLC (Agilent, USA) with a binary pump, a degasser, autosampler and a DAD UV detector; Agilent 1200 High Performance Liquid Chromatographiy (HPLC) (Agilent, USA) with a binary pump, a degasser, Autosampler and a DAD detector, All the operations and the data acquiring were controlled by a Agilent chemstation software.

The separation of eight polyphenols was optimized and performed on an Agilent Eclipse XDB- $\mathrm{C}_{18}$ RRLC column, $50 \times 4.6 \mathrm{~mm}$ id $(1.8 \mu \mathrm{m}$ pore size, Agilent, USA). The mobile phase consisted of (A) $0.1 \%$ formic acid aqueous solution and (B) acetonitrile. The solvent program was initially 2 min isocratic with $90 \% \mathrm{~A}$ and $10 \% \mathrm{~B}$, then from 2 to $5 \mathrm{~min}$ linear gradient to $30 \% \mathrm{~B}$, finally at $6.5 \mathrm{~min}$ linear gradient to $10 \% \mathrm{~B}$ and re-equilibrium for additional $1.5 \mathrm{~min}$ for subsequent analysis. The flow rate was $2.0 \mathrm{~mL} / \mathrm{min}$. The detection wavelength was set at $340 \mathrm{~nm}$. The injection volume was $5 \mu \mathrm{L}$. The column temperature was set at $30^{\circ} \mathrm{C}$. Thus, the total time for one-run chromatographic separation was not more than $8 \mathrm{~min}$.

\subsection{Tobacco Sample Pretrement}

The tobacco samples were prepared in our laboratory as follow. One hundred grams of the fluecured tobacco leaves (without peduncle) were chopped and crushed to produce the tobacco powder at 40 mesh. $0.25 \mathrm{~g}$ of tobacco powder was extracted with ultrasonic extraction for $30 \mathrm{~min}$ in $40 \mathrm{~mL}$ of methanol-water $(70: 30, \mathrm{v} / \mathrm{v})$ solution and centrifuged at $10,000 \mathrm{rpm}$ for $10 \mathrm{~min}$. Afterwards, $5 \mathrm{~mL}$ solution of centrifuge was loaded onto a Waters Sep-Park- $\mathrm{C}_{18}$ cartridge (500 mg) previously conditioned with $10 \mathrm{~mL}$ methanol and $10 \mathrm{~mL}$ deionized water, respectively. The first $3 \mathrm{~mL}$ eluates were discarded, the following $2 \mathrm{~mL}$ eluates were collected and filtered through a $0.22 \mu \mathrm{m}$ membrane. The tobacco extract was directly analyzed by RRLC. 


\section{Results and Discussion}

\subsection{Optimization of RRLC Conditions}

The separation of eight polyphenols was initially performed on a ZORBAX Eclipse XDB- $\mathrm{C}_{18}$ column $(150 \times$ $4.6 \mathrm{~mm}$ id, $5 \mu \mathrm{m}$ ) by conventional HPLC. Although they were achieved the batter separation under the optimized HPLC conditions, the analysis time was more than 20 min (Figure 2(a)). It is not suitable for the throughput analysis. Thus, a new RRLC separation system was chosen. Using the Eclipse XDB- $\mathrm{C}_{18}$ RRLC column $(50 \times 4.6$ $\mathrm{mm}$ i.d., $1.8 \mu \mathrm{m})$, different mobile phase, elution program, flow rate and column temperature were investigated. For example, at flow rate $1.0 \mathrm{~mL} / \mathrm{min}$ and column temperature $20^{\circ} \mathrm{C}$, The longest retention time was less than $6 \mathrm{~min}$, however SP and RT can not be resolved (Figure 2(b)). By increasing the flow rate from 1 to 2

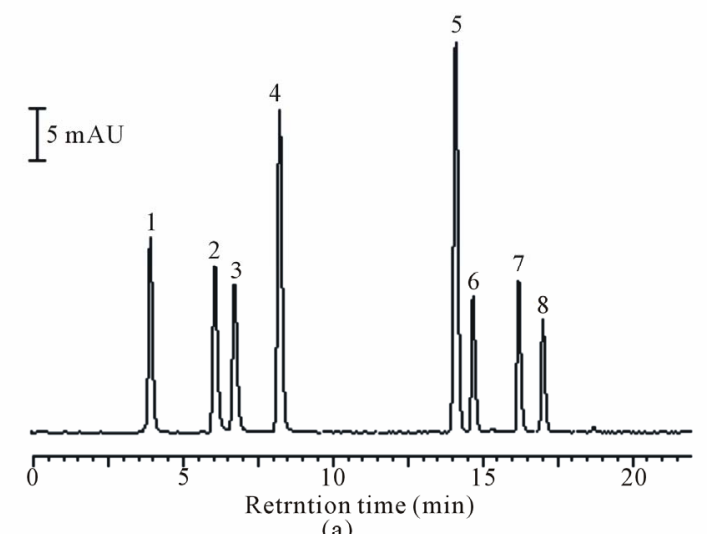

(a)

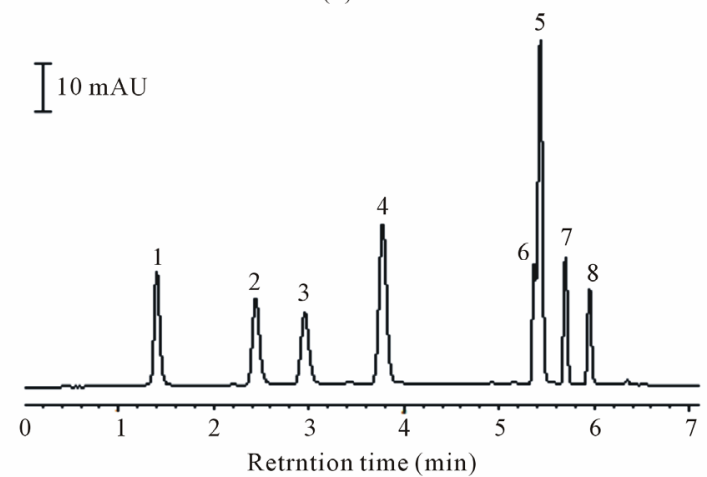

(b)

Figure 2. Separations of eight polyphenols by (a) HPLC and (b) RRLC at $20^{\circ} \mathrm{C}$. (a) HPLC column: Eclipse XDB-C18 $150 \times 4.6 \mathrm{~mm}$ i.d., $5 \mathrm{\mu m}$; Mobile A: $0.1 \%$ formic acid $/ \mathrm{H}_{2} \mathrm{O}$; $B$ : cetonitrile; Flow rate: $1.0 \mathrm{~mL} / \mathrm{min}$; Temperature: $20^{\circ} \mathrm{C}$. (b) RRLC column: Eclipse XDB-C18 $50 \times 4.6 \mathrm{~mm}$ i.d., 1.8 $\mu \mathrm{m}$; Mobile A: 0.1\% formic acid $/ \mathrm{H}_{2} \mathrm{O}$; B: acetonitrile; Flow rate: $1.0 \mathrm{~mL} / \mathrm{min}$; Temperature: $20^{\circ} \mathrm{C}$; Peaks: 1 , 5-O-caffioylquinic acid; 2 , chlorogenic acid; 3 , 4-o-caffioylquinic acid; 4, caffeic acid; 5, scopoletin; 6, rutin; 7, kaempferol3-rutinoside; 8, quercitrin.
$\mathrm{mL} / \mathrm{min}$ and column temperature from $20^{\circ} \mathrm{C}$ to $30^{\circ} \mathrm{C}$, eight polyphenols were separated in baseline within 5 min (Figure 3(a)). More importantly, the analysis was faster 3 times than conventional HPLC without sacrificing resolution. micellar liquid chromatography (MLC) also can separate and quantify 3 tobacco polyphenols (chlorogenic acid, rutin and scopoletin) in less than 10 min, but the theoretical plate of chlorogenic acid in tobacco sample using RRLC (2116 plates) was much higher than that using MLC (576 plates).

\subsection{Choice of Extraction and Cleanup Method}

To extract polyphenols from tobacco leaf powder, the following three methods were compared using $40 \mathrm{~mL}$ methanol/ water $(70: 30, \mathrm{v} / \mathrm{v})$ as extraction solvent for $0.2500 \mathrm{~g}$ tobacco leaves: 1) refluxed at $60^{\circ} \mathrm{C}$ for $40 \mathrm{~min}$; 2) ultrasonic extraction under $50 \mathrm{~Hz}$ for $40 \mathrm{~min}$; and 3)

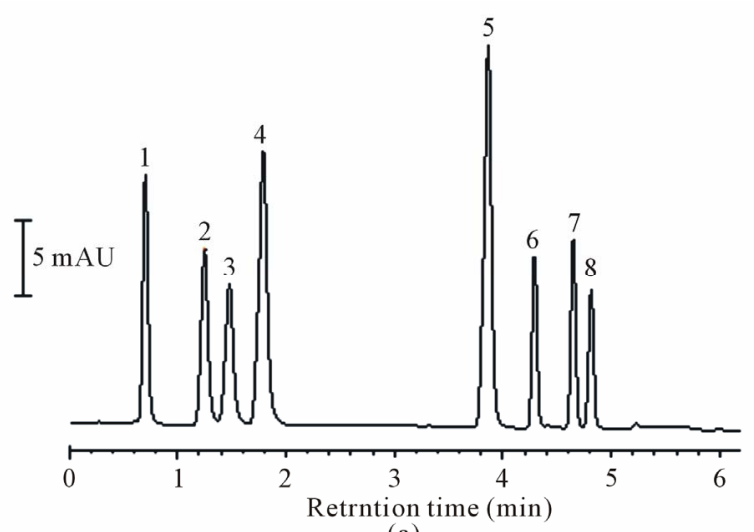

(a)

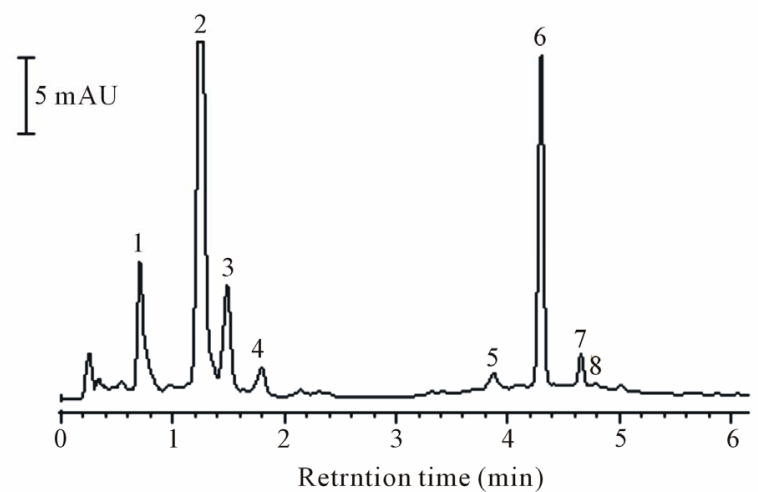

(b)

Figure 3. Chromatograms for (a) polyphenol standards and (b) tobacco samples by RRLC RRLC column: Eclipse XDB-C18 $50 \times 4.6 \mathrm{~mm}$ i.d., $1.8 \mu \mathrm{m}$; Mobile A: $0.1 \%$ formic acid/ $\mathrm{H}_{2} \mathrm{O}$; B: acetonitrile; Gradient: 0 min (10\% B) $\sim 2.0$ $\min (15 \% B \sim 5.0 \mathrm{~min}(30 \% \mathrm{~B}) \sim 6.5 \mathrm{~min}(50 \% \mathrm{~B}) \sim 8.0 \mathrm{~min}$ $(10 \% \mathrm{~B})$; Flow rate: $2.0 \mathrm{~mL} / \mathrm{min}$; Temperature: $30^{\circ} \mathrm{C}$. Peaks: 1, 5-o-caffioylquinic acid; 2, chlorogenic acid; 3 , 4-O-caf-fioylquinic acid; 4, caffeic acid; 5, scopoletin; 6, rutin; 7, kaempferol-3-rutinoside; 8, quercitrin. 
mechanical shaking extraction under 15 cycles per minute for $40 \mathrm{~min}$. The results indicated that the extraction efficiency of refluxed extraction and ultrasonic extraction (Recoveries of eight polyphenols $>90 \%$ ) were better than mechanical shaking extraction (Recovery of Scopoletin $<80 \%$ ). Because ultrasonic extraction is simpler than two others, it was chosen for the polyphenols extraction in this paper.

SPE has been proven to be an effective tool for selectively removing interferences, enabling sensitive, selective and robust analysis. There are some weak polar materials in tobacco extract which can not be removed completely from $\mathrm{C}_{18}$ column by mobile phase, such as fatty substance, wax, pigment, and so on. Moreover, the smaller stationary phase particles make it easier plug for RRLC than HPLC. So it is necessary to clean up the extract before RRLC analysis. In this paper, the solution of extraction was clean up with a Waters Sep-Park $\mathrm{C}_{18}$ cartridge, by which the weak polar material was retained, and the ployphenol fractions were collected for RRLC analysis.

\subsection{Linearity of Calibration and Limit of Detection for Eight Ployphenols}

Using the optimized RRLC condition, the linear ranges of the UV response at $340 \mathrm{~nm}$ were observed over the concentration range from 0.5 to $120 \mu \mathrm{g} / \mathrm{mL}$ for eight ployphenols. The regressions between peak area (y) and concentration $(\mathrm{x}, \mu \mathrm{g} / \mathrm{ml})$ yielded the linear equations as decribed in Table 1.

The limit of detection (LOD) was evaluated by calculating a signal to noise ratio of $3(\mathrm{~S} / \mathrm{N}=3)$. The result was summarized in Table 1.

From Table 1, it can be seen that the LODs by RRLC were lower than those by HPLC, which enhanced the detection sensitivity for eight ployphenols.

\subsection{Method Validation}

A series of samples analysis were performed to validate the performance of the method. The accuracy was assessed with recovery assay by adding eight standard polyphenols to sample at low and high levels. The recovery was calculated by comparing the found mount of standards to those of added. The precision was evaluated from replicated determinations $(n=5)$ performed on the different day for same samples. The recoveries of eight polyphenols ranged from $94 \%$ to $107 \%$ with the relative standard deviation (RSD) less than $2.0 \%$.

\subsection{Application to Real Tobacco Samples}

The proposed RRLC method was used for routine analysis of four tobacco samples from different areas. A typical chromatogram was displayed in Figure 3(b) and the results obtained were summarized in Table 2 . The results are consistent with those determined by Zhang [16] and

Table 1. Regression equation and the LODs.

\begin{tabular}{ccccc}
\hline Compound & Regression equation & Correlation coefficient & LOD/RRLC $(\mathrm{ng} / \mathrm{mL})$ & LOD/HPLC $(\mathrm{ng} / \mathrm{mL})$ \\
\hline NG & $\mathrm{y}=1.9951 \mathrm{x}-0.5391$ & $\mathrm{R}^{2}=0.9994$ & 59.4 & 128.2 \\
CG & $\mathrm{y}=1.8874 \mathrm{x}-0.9993$ & $\mathrm{R}^{2}=0.9996$ & 105.3 & 211.2 \\
YG & $\mathrm{y}=1.7039 \mathrm{x}-0.6706$ & $\mathrm{R}^{2}=0.9999$ & 103.5 & 178.6 \\
CA & $\mathrm{y}=3.5595 \mathrm{x}-0.9966$ & $\mathrm{R}^{2}=0.9991$ & 51.3 & 69.4 \\
SP & $\mathrm{y}=4.2813 \mathrm{x}+0.1804$ & $\mathrm{R}^{2}=0.9995$ & 30.3 & 43.0 \\
RT & $\mathrm{y}=1.2785 \mathrm{x}+0.2096$ & $\mathrm{R}^{2}=0.9995$ & 66.7 & 93.8 \\
KR & $\mathrm{y}=1.3913 \mathrm{x}+0.3109$ & $\mathrm{R}^{2}=0.9995$ & 58.8 & 84.7 \\
QT & $\mathrm{y}=1.0483 \mathrm{x}+0.1352$ & $\mathrm{R}^{2}=0.9995$ & 84.5 & 121.0 \\
\hline
\end{tabular}

Table 2. The determination results of eight polyphenols in tobacco samples.

\begin{tabular}{|c|c|c|c|c|}
\hline Compounds & Flue-cured tobacco (Yunnan) & Burley tobacco (Hubei) & Oriental tobacco (Yunnan) & Zimbabwel \\
\hline NG & $0.247 \%$ & $0.008 \%$ & $0.156 \%$ & $0.203 \%$ \\
\hline $\mathrm{CG}$ & $1.522 \%$ & $0.024 \%$ & $0.666 \%$ & $1.156 \%$ \\
\hline YG & $0.361 \%$ & $0.013 \%$ & $0.289 \%$ & $0.263 \%$ \\
\hline $\mathrm{CA}$ & $0.016 \%$ & $0.007 \%$ & $0.016 \%$ & $0.025 \%$ \\
\hline $\mathrm{SP}$ & $0.017 \%$ & $0.005 \%$ & $0.008 \%$ & $0.032 \%$ \\
\hline $\mathrm{KR}$ & $0.060 \%$ & $0.004 \%$ & $0.080 \%$ & $0.049 \%$ \\
\hline QT & $0.022 \%$ & unfound & $0.014 \%$ & $0.020 \%$ \\
\hline
\end{tabular}


Li [17]. The method demonstrated that it is usable and applicable to the rapid and sensitive determination of polyphenols in tobacco.

\section{Conclusions}

In this paper, a simple, fast, sensitive and reproducible RRLC analytical method for for eight polyphenols in the tobacco samples was developed by coupling with a practical sample pretreatment. With ultrasonic extraction and Solid-phase extraction (SPE), higher recoveries were obtained with lower matrix interfering for RRLC separation of eight polyphenols. The developed method was suitable for high throughput analysis of the tobacco samples.

\section{Acknowledgements}

The authors gratefully acknowledge for the financial support of the National Nature Science Foundation of China (No. 20875083, 21077095), the Scientific Foundation of the State Tobacco Monopoly Administration of China, and the Innovation Scientists \& Technicians Troop Construction Projects of Zhengzhou City (10LJRC192). They also thank Dr. Julie Rimes for assistance with editing the text.

\section{References}

[1] T. J. Zuo, "Production, Physicology and Biochemistry of Tobacco," Shanghai Far East Press, Shanghai, 1993.

[2] L. Bazinet, Y. De Grandpre and A. Porter, "Electromigration of Tobacco Polyphenols," Separation and Purification Technology, Vol. 41, No. 1, 2005, pp. 101-107. doi:10.1016/j.seppur.2004.05.003

[3] C. Gong, A. Wang and S. Wang, "Changes of Polyphenols in Tobacco Leaves during the Flue-Curing Process and Correlation Analysis on Some Chemical Components," Agricultural Sciences in China, Vol. 12, 2006, p. 928932.

[4] L. Bazinet, Y. De Grandpre and A. Porter, "Enhanced Tobacco Polyphenol Electromigration and Impact on Membrane Integrity," Journal of Membrane Science, Vol. 254, No. 1-2, 2005, pp. 111-118. doi:10.1016/i.memsci.2004.11.029

[5] F. J. C. Roe, M. H. Salaman and J. Cohen, "Incomplete Carcinogens in Cigarette Smoke Condensate: Tumourpromotion by a Phenolic Fraction," British Journal of Cancer, Vol. 13, 1959, pp. 623-633. doi: $10.1038 /$ bjc. 1959.68

[6] S. J. Sheen, "Colorimetric Determination of Chlorogenic Acid and Rutin in Tobacco Leaves," Tobacco Science,
Vol. 15, 1971, pp. 116-120.

[7] F. Li, Q. Liu, W. Cai and X. G. Shao, "Analysis of Scopoletin and Caffeic Acid in Tobacco by GC-MS after a Rapid Derivatization Procedure," Chromatographia, Vol. 69, No. 7-8, 2009, pp. 743-748. doi:10.1365/s10337-008-0938-2

[8] H. Cui, C. He and G. Zhao, "Determination of Polyphenols by High-Performance Liquid Chromatography with Inhibited Chemiluminescence Detection," Journal of Chromatography A, Vol. 855, No. 1, 1999, pp. 171-179. doi:10.1016/S0021-9673(99)00670-6

[9] Y. Chen, Q. J. Yu, X. Li, Y. Luo and H. Liu, "Extraction and HPLC Characterization of Chlorogenic Acid from Tobacco Residuals," Separation Science and Technology, Vol. 42, No. 15, 2007, pp. 3481-3492. doi:10.1080/01496390701626677

[10] J. Gu, X. Zeng, B. Kong, Y. Mao, W. Liu and W. Wei, "Rapid Determination of Polyphenols in Tobacco by MLC," Chromatographia, Vol. 71, No. 9-10, 2010, pp. 769-774. doi:10.1365/s10337-010-1533-x

[11] H. Wang, M. Zhao, B. Y. Yang and G. Jiang, "Identification of Polyphenols in Tobacco Leaf and Their Antioxidant and Antimicrobial Activities," Food Chemistry, Vol. 107, No. 4, 2008, pp. 1399-1406. doi:10.1016/j.foodchem.2007.09.068

[12] H. Jiang, Y. He, H. Zhao and Y. Hu, "Determination of Chlorogenic Acid and Rutin in Cigarettes by an Improved Capillary Electrophoresis Indirect Chemiluminescence System," Analytica Chimica Acta, Vol. 512, No. 1, 2004, pp. 111-119. doi:10.1016/j.aca.2004.02.021

[13] A. Garrido Frenich and J. L. Martínez Vidal, "HighThroughput Determination of Pesticide Residues in Food Commodities by Use of Ultra-Performance Liquid Chromatography-Tandem Mass Spectrometry," Analytical and Bioanalytical Chemistry, Vol. 389, No. 6, 2007, pp. 1-14.

[14] M. Barreda, J. V. Sancho and F. Hernández, "Multiresidue Pesticide Analysis of Fruits by Ultra-Performance Liquid Chromatography Tandem Mass Spectrometry," Analytical and Bioanalytical Chemistry, Vol. 389, 2007, pp. 1765-1771. doi:10.1007/s00216-007-1407-8

[15] R. Batlle, J. Romero and C. Nerín, "UPLC-MS as a Powerful Technique for Screening the Nonvolatile Contaminants in Recycled PET," Analytical and Bioanalytical Chemistry, Vol. 388, No. 5-6, 2007, pp. 1031-1038. doi:10.1007/s00216-007-1341-9

[16] T. Zhang, X. Dong and F. Wu, "Microcolumn High Performance Liquid Chromatography and Spectrometry Method for the Determination of Polyphenols in Tobacco," Chinese Journal Instrumental Analysis, Vol. 23, 2004, pp. 274-275.

[17] F. Li, W. Cai and X. Shao, "Analysis of Polyphenols in Tobacco Using Reversed-Phase HPLC," Chinese Journal of Chromatography, Vol. 25, No. 4, 2007, pp. 565-585. 